\title{
Kondo peaks and dips in the differential conductance of a multi-lead quantum dot: Dependence on bias conditions
}

\author{
M. Ţolea ${ }^{1}$, I. V. Dinu ${ }^{1}$, and A. Aldea ${ }^{1,2}$ \\ 1 National Institute of Materials Physics, POB MG-7, 77125 Bucharest-Magurele, Romania. \\ 2 Institute of Theoretical Physics, Cologne University, 50937 Cologne, Germany.
}

\begin{abstract}
We study the differential conductance in the Kondo regime of a quantum dot coupled to multiple leads. When the bias is applied symmetrically on two of the leads ( $V$ and $-V$, as usual in experiments), while the others are grounded, the conductance through the biased leads always shows the expected enhancement at zero bias. However, under asymmetrically applied bias $(V$ and $\lambda V$, with $\lambda>0$ ), a suppression - dip - appears in the differential conductance if the asymmetry coefficient $\lambda$ is beyond a given threshold $\lambda_{0}=\sqrt[3]{1+r}$ determined by the ratio $r$ of the dot-leads couplings. This is a recipe to determine experimentally this ratio which is important for the quantum-dot devices. This finding is a direct result of the Keldysh transport formalism. For the illustration we use a many-lead Anderson Hamiltonian, the Green functions being calculated in the Lacroix approximation, which is generalized to the case of nonequilibrium.
\end{abstract}

\section{INTRODUCTION}

The observation of the Kondo effect in quantum dots allows a direct insight on this interesting many-body effect, whose experimental fingerprint is the enhanced differential conductance at zero bias which is a specific type of zero bias anomaly. The great advantage over the bulk Kondo effect is of course the possibility to control the parameters like for instance the coupling with the leads. Next, it was shown that even more information can be extracted if the dot is connected to three leads. The three lead-Kondo problem was theoretically considered before in, e.g., 3, 4, 5] and experimentally realized by Leturcq et. al [6, 7]. The third lead was used to read the non-equilibrium density of states (DOS) induced by the other two leads. Shah and Rosch [5] analyzed the influence of the coupling and bias on the decoherence rate.

In this paper, we also study the many-lead quantum dots in Kondo regime, but the focus is different : we are interested to find out the conditions under which the differential conductance can show a suppression at zero bias instead of the usual enhancement. There are well-known examples of transport properties influenced by dips in the spectral function produced by Coulomb interactions. The first one was the Coulomb pseudo-gap in the impurity conduction of doped semiconductors resulting in the $T^{-1 / 2}$ dependence of the hopping conductivity [1]. Then, a zero bias anomaly manifested as a dip in tunneling DOS was studied in tunnel junctions [2]. However, what is conspicuous for the three-lead quantum dots (see sketch in Fig.2), is that a peak-dip crossover may appear in the differential conductance, while keeping the dot permanently in Kondo regime. As we show in the next section, this occurs when the bias is applied asymmetrically on the left and right leads, and the crossover is triggered by the degree of asymmetry. For the sake of definiteness, let us assume that for some voltage configuration (see sketch in Fig.2), the left lead receives electrons from the central lead and pumps electrons into the right lead, the difference of the two contributions being the total current. For the conductances, one can write $\mathcal{G}_{L}=\mathcal{G}_{L C}+\mathcal{G}_{L R}=\mathcal{G}_{L C}-\left|\mathcal{G}_{L R}\right|$. Both $\mathcal{G}_{L C}$ and $\left|\mathcal{G}_{L R}\right|$ must decay by applying a voltage $V$ (as the voltage destroys the Kondo resonance and reduces the electronic tunneling between leads), but not necessarily with the same derivative ; then, if $d \mathcal{G}_{L C} / d V>d\left|\mathcal{G}_{L R}\right| / d V$ the conductance $\mathcal{G}_{L}$ will increase with $V$, giving rise to a dip about $V=0$.

It will also be shown that the peak-dip crossover can be used to determine the ratio between the couplings of different leads to the dot, and therefore it is of potential experimental use.

The main conclusions of the paper are extracted from the current formula given by the Keldysh formalism (Eq.1) by taking advantage of a simple connection between the convexity of the conductance $d I / d V$ and the convexity of the density of states.

Recently, in the first two-channel Kondo experiment [8], a dip generated by the competition between channels was observed in the differential conductance, even in the case of symmetrically applied bias. One may note that in our calculations, as in the mentioned (theoretical and experimental) papers referring to the 3-lead problem [3, 5, 6, 7], the set-up and physics are different, and therefore the results are outside the multi-channel Kondo phenomenology.

For the illustration of the results, we shall give also some numerical calculations using the equation of motion solution for the Anderson Hamiltonian [9], generalized to the multi-lead and non-equilibrium case. 


\section{PEAK-DIP CROSSOVER IN DIFFERENTIAL CONDUCTANCE: ANALYTICAL ANALYSIS}

In order to calculate the electron current through the lead $\alpha$ one can use the Keldysh formalism, and obtain [3]:

$$
I_{\alpha}=-\frac{4 e \Delta_{\alpha}}{\pi \hbar \Delta} \int_{-\infty}^{\infty} \operatorname{Im}_{d d}(\omega) \sum_{\beta(\neq \alpha)} \Delta_{\beta}\left[f\left(\omega-\mu_{\alpha}\right)-f\left(\omega-\mu_{\beta}\right)\right] d \omega, \quad \sum_{\alpha} I_{\alpha}=0,
$$

where $\Delta_{\alpha}$ is the coupling of the lead $\alpha$ to the dot, $\Delta=\sum \Delta_{\alpha}$, and $f\left(\omega-\mu_{\alpha}\right)$ is the Fermi distribution in the $\alpha$ lead. $G_{d d}$ is the retarded Green function of the dot in the presence of the leads. We remind that, usually, the Keldysh formalism needs also the "lesser" Green function, but when the leads are all connected in the same site, the lesser function can be eliminated (except for the case of magnetic leads [10]). It was recently proven that the same simplification occurs for a symmetric many-site system [11].

In this paper we are interested in the behavior of the conductance close to equilibrium, for the case when two of the leads are biased and the others are grounded. In this case, all the grounded leads have the same chemical potential (set to zero) and act like one single reservoir; this reservoir will be called "central lead" (with the chemical potential $V_{C}=0$ ). If we apply the potential $V_{L}=V$ on the left lead " $L$ " and the potential $V_{R}=\lambda V$ on the right lead " $R$ ", then the differential conductance through the lead " $L$ " can be calculated from Eq.1 :

$$
\mathcal{G}_{L}(V)=\frac{d I_{L}}{d V}=\frac{4 e^{2} \Delta_{L}}{\pi \hbar \Delta} \int_{-\infty}^{\infty}\left[\left(\Delta_{R}+\Delta_{C}\right) \operatorname{Im} G_{d d}(\omega) \frac{d f(\omega-e V)}{d \omega}-\lambda \Delta_{R} \operatorname{Im} G_{d d}(\omega) \frac{d f(\omega-\lambda e V)}{d \omega}\right] d \omega .
$$

Notice that only the derivative of the Fermi function was considered while the derivative of $\operatorname{Im} G_{d d}$ versus bias was neglected, which was proven to be a very good approximation in [3]. At low temperatures, when the derivatives of the Fermi functions approximate delta functions, the above formula turns into :

$$
\mathcal{G}_{L}(V)=-\frac{4 e^{2} \Delta_{L}}{\pi \hbar \Delta}\left[\left(\Delta_{R}+\Delta_{C}\right) \operatorname{Im} G_{d d}(e V)-\lambda \Delta_{R} \operatorname{Im} G_{d d}(\lambda e V)\right]
$$

and similarly:

$$
\begin{aligned}
& \mathcal{G}_{R}(V)=\frac{d I_{R}}{d V}=-\frac{4 e^{2} \Delta_{R}}{\pi \hbar \Delta}\left[\lambda\left(\Delta_{L}+\Delta_{C}\right) \operatorname{Im} G_{d d}(\lambda e V)-\Delta_{L} \operatorname{Im} G_{d d}(e V)\right] \\
& \mathcal{G}_{C}(V)=\frac{d I_{C}}{d V}=-\frac{4 e^{2} \Delta_{C}}{\pi \hbar \Delta}\left[-\Delta_{L} \operatorname{Im} G_{d d}(e V)-\lambda \Delta_{R} \operatorname{Im} G_{d d}(\lambda e V)\right]
\end{aligned}
$$

The conductance $\mathcal{G}_{L, R, C}$, defined in this way is a quantity that can be measured experimentally and helps for determining the coupling ratios as we shall see below.

We suggest now a way to search for Kondo dips in the differential conductance. One has to compute the second derivative of $\mathcal{G}_{L, R, C}$ for $V=0$ which contains the convexity information (in case the first derivative vanishes, the convexity determines if one has a peak or a dip). The second derivative of the differential conductance can be expressed as:

$$
\begin{array}{r}
\mathcal{G}_{L}{ }^{\prime \prime}(0)=-\frac{4 e^{4} \Delta_{L} \Delta_{R}}{\pi \hbar \Delta} \operatorname{Im} G_{d d}^{\prime \prime}(0)\left[\left(1+\frac{\Delta_{C}}{\Delta_{R}}\right)-\lambda^{3}\right] \\
\mathcal{G}_{R}{ }^{\prime \prime}(0)=-\frac{4 e^{4} \Delta_{L} \Delta_{R}}{\pi \hbar \Delta} \operatorname{Im} G_{d d}^{\prime \prime}(0)\left[\left(1+\frac{\Delta_{C}}{\Delta_{R}}\right) \lambda^{3}-1\right] \\
\mathcal{G}_{C}^{\prime \prime}(0)=\frac{4 e^{4} \Delta_{C} \Delta_{R}}{\pi \hbar \Delta} \operatorname{Im} G_{d d}^{\prime \prime}(0)\left[\frac{\Delta_{L}}{\Delta_{R}}+\lambda^{3}\right] ;
\end{array}
$$

In the above formulas $-\operatorname{Im} G_{d d}^{\prime \prime}(0)$ is the second derivative of the spectral function at equilibrium (i.e. for $V=0$ ) and is always negative for $T<T_{K}$ indicating the presence of the Kondo peak in the density of states. This property will be used to determine the peak or dip behavior of the conductance.

The distribution (and sign) of the conductances in the three-lead device is controlled by the coupling to the ground lead $\Delta_{C}$. In the mentioned experiments [6, , 7] , when the aim was to read the non-equilibrium DoS, $\Delta_{C}$ is very small compared to the other couplings; in this case the currents L and R have always opposite signs, the same being true for the second derivatives (6-7); the conclusion is that both conductances are maximum in module at zero bias. However, for large coupling $\Delta_{C}$ the above formulae indicate the possibility of a more complex behavior of the conductance.

We identify first the case $\lambda=-1$, as being the one commonly used, with the gate potentials symmetrically applied. In Eq.6, the straight parenthesis on the r.h.s is always positive for negative $\lambda$, so one may conclude that $\mathcal{G}_{L}{ }^{\prime \prime}(0)$ has the 
same sign as $-\operatorname{Im} G_{d d}^{\prime \prime}(0)$, which is negative, indicating a peak also in the differential conductance $\mathcal{G}_{L}$. For negative $\lambda$, it turns out from Eqs.3,4 that $\mathcal{G}_{L}>0$ and $\mathcal{G}_{R}<0$, meaning that the left and right currents flow in opposite directions. Then the above argument holds for the module of $\mathcal{G}_{R}$ which is also maximum at zero bias.

Interestingly, for $\Delta_{L}=\Delta_{R}$ and $\lambda=-1, \mathcal{G}_{C}$ is not zero as a consequence of the spectral asymmetry $\operatorname{Im} G_{d d}(e V) \neq$ $\operatorname{Im} G_{d d}(-e V)$ (see Eq.5, and also notice the different heights of the side peaks in Fig.1). The same non-zero value of the current through the grounded lead was discussed in [12], for the sequential tunneling case, and attributed to the electron-hole asymmetry. Under the above mentioned conditions $\mathcal{G}_{C}{ }^{\prime \prime}(V=0)=0$ indicating a saddle point. In this case, the conductance through the central lead does not show a peak shape, being instead antisymmetrical about $V=0$.

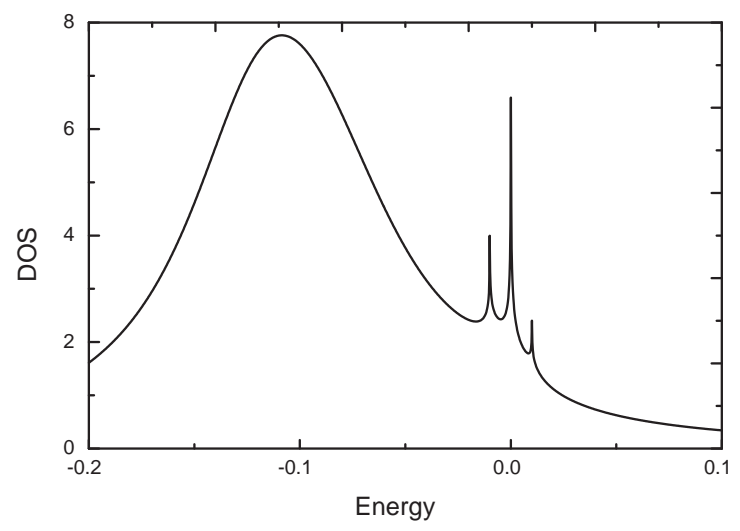

FIG. 1: The dot density of states for the case of symmetrically applied bias $(\lambda=-1)$. The three Kondo peaks correspond to the chemical potentials in the three leads. Notice that the side peaks are different in height. The parameters are: $V=0.01$, $\epsilon_{d}=-0.12, \Delta_{R}=\Delta_{L}=0.01, \Delta_{C}=0.02 \Delta_{C} / \Delta_{L}=2, \Delta_{R}=\Delta_{L}=0.01$. The temperature is $T=T_{K} / 10$, and $T_{K}=3.2 \cdot 10^{-4}$.
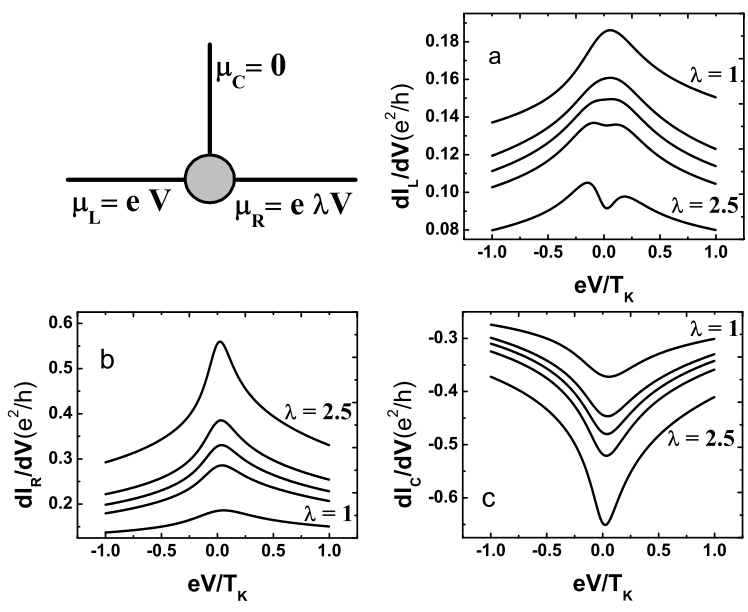

FIG. 2: Sketch of a quantum dot connected to three leads, with the bias asymmetrically applied on the left and right leads ( $V$ and $\lambda V$, with $\lambda=1 / 1.4 / 1.58 / 1.8 / 2.5$ ). (a) The differential conductance through the left lead changes from peak to dip (around zero bias). The value of the asymmetry parameter $\lambda$ where the crossover happens is $\lambda_{0}=\sqrt[3]{1+\Delta_{C} / \Delta_{L}}=1.58$. (b,c) Differential conductance through the right and central lead show a Kondo peak behavior being maximum in module at $V=0$ The other parameters : $\Delta_{R}=\Delta_{L}=0.075, \Delta_{C} / \Delta_{L}=3, \epsilon_{d}=-0.12, T=T_{K} / 10$, and $T_{K}=3.2 \cdot 10^{-4}$.

A different situation occurs in the case $\lambda>0$. It is obvious that, without loss of generality, we may consider actually $\lambda \geq 1$ meaning that, by convention, the smaller bias is applied on the left lead. One may now conclude from Eq.6 
that the peak/dip behavior in the left lead is completely determined by the sign of $\left(1+\Delta_{C} / \Delta_{R}\right)-\lambda^{3}$. This result may be of potential interest for experimentalists. In particular, if one finds the crossover value $\lambda_{0}=\sqrt[3]{1+\Delta_{C} / \Delta_{R}}$ (when the peak changes into dip), the ratio $\Delta_{C} / \Delta_{R}$ can be immediately extracted. The mentioned coupling ratio is otherwise inaccessible to direct measurement. Such a peak-dip crossover can be seen in the Fig.2a. The numerical plot (see below calculation details) indicates that the dip is considerably narrower than the Kondo temperature, and is situated in the middle of a larger peak. This means that even if the conductance through the left lead initially increases, it will eventually start to decrease for a larger bias. It is an expected behavior, since for large biases all Kondo correlations are killed and all the conductances approach the (much lower) non-Kondo limit.

The conductances through the right and central leads have the usual peak behavior (see Fig.2b,c). One has to pay attention to the fact that the conductance through the central lead is negative due to the sign convention regarding the currents direction, but it is maximum in module at zero bias, and should be considered as a Kondo peak.

We stress that the formulas Eqs.6-8 are quite general, and also hold outside the Kondo regime (for high temperatures or weak couplings), but in this case they are not very insightful. The variation of the differential conductance is very smooth outside the Kondo regime, and it is difficult to determine its convexity unambiguously.

\section{APPLICATION: NUMERICAL RESULTS FOR THE MULTI-LEAD ANDERSON MODEL}

In order to illustrate the results, we shall calculate the differential conductance for the multi-lead Anderson model:

$$
H=\sum_{\sigma} \epsilon_{d} d_{\sigma}^{\dagger} d_{\sigma}+U d_{\uparrow}^{\dagger} d_{\uparrow} d_{\downarrow}^{\dagger} d_{\downarrow}+\sum_{\alpha, k, \sigma} \epsilon_{k \sigma} c_{k \alpha \sigma}^{\dagger} c_{k \alpha \sigma}+\sum_{\alpha, k, \sigma} V_{\alpha}\left(c_{k \alpha \sigma}^{\dagger} d_{\sigma}+H . c .\right),
$$

where $\epsilon_{d}$ is the energy of the dot level, $U$ is the Coulomb repulsion and, for instance, $c_{k \alpha \sigma}$ annihilates an electron with momentum $k$ and spin $\sigma$ in the $\alpha$ lead. The last term describes the coupling between the dot and leads. The quantity needed in the current formula Eq.1 is the dot retarded Green function $G_{d d, \sigma}=<<d_{\sigma}, d_{\sigma}^{\dagger}>>$, which will be calculated in the Lacroix [9] approach. We write the equation of motion for the Green function and perform a decoupling approximation at the third step in order to close the system of equations. In our case, we consider three leads (reservoirs) instead of one as in [9], the generalization being straightforward. The resulting formula, in the $U \rightarrow \infty$ limit, reads:

$$
G_{d d, \sigma}(\omega+i 0)=\frac{1-\frac{\leq n_{d}>}{2}-\sum_{k, \alpha} V_{\alpha} \frac{\left\langle d_{-\sigma}^{\dagger} c_{k \alpha-\sigma}>\right.}{\omega-\epsilon_{k}}}{\omega-E_{0}-\sum_{k, \alpha} \frac{V_{\alpha}^{2}}{\omega-\epsilon_{k}}-\sum_{k, q, \alpha, \beta} V_{\alpha} V_{\beta} \frac{\left\langle c_{q \alpha-\sigma}^{\dagger} c_{k \beta-\sigma}>\right.}{\omega-\epsilon_{k}}+\sum_{k, \alpha} \frac{V_{\alpha}^{2}}{\omega-\epsilon_{k}} \sum_{k, \alpha} V_{\alpha} \frac{\left\langle d_{-\sigma}^{\dagger} c_{k \alpha-\sigma}>\right.}{\omega-\epsilon_{k}}}
$$

Since we work in a non-magnetic case, the subscript $\sigma$ can be omitted in the Green function notation. It comes now into question how to treat the averages of the type $\left\langle c_{q \alpha-\sigma}^{\dagger} c_{k \beta-\sigma}\right\rangle$, for instance, where the two operators refer to electrons in different leads. For equilibrium, when all the Fermi levels are equal, one can use the fluctuation-dissipation theorem which relates the average to the corresponding Green function:

$$
<c_{q \alpha-\sigma}^{\dagger} c_{k \beta-\sigma}>=-\frac{1}{\pi} \int f(\omega) \operatorname{Im}<<c_{k \beta-\sigma} c_{q \alpha-\sigma}^{\dagger}>>d \omega,
$$

In non-equilibrium, the Fermi functions $f_{\alpha}$ are different in the three leads $\alpha=L, R, C$, and for the calculation of the mentioned average we propose

$$
<c_{q \alpha-\sigma}^{\dagger} c_{k \beta-\sigma}>=-(1 / 2 \pi) \int\left(f_{\alpha}(\omega)+f_{\beta}(\omega)\right) \operatorname{Im}<<c_{k \beta-\sigma} c_{q \alpha-\sigma}^{\dagger}>>d \omega .
$$

In the calculations there also appear averages of the type $\left\langle d_{-\sigma}^{\dagger} c_{k \alpha-\sigma}\right\rangle$, so that a distribution function on the dot is also needed. For the dot we shall use the weighted mean $f_{d}=\sum_{\alpha} \Delta_{\alpha} f_{\alpha} / \Delta$. This intuitive expression of $f_{d}$ has been used in, e.g. [13, 14], and we have checked that the present results, i.e. existence of the dip and its temperature dependance, are stable with respect to different ways to introduce the non-equilibrium.

These non-equilibrium aspects being settled, we continue along the Lacroix line, and with the definitions $\Delta=$ $\sum \Delta_{\alpha}=i \sum_{k, \alpha} \frac{V_{\alpha}^{2}}{\omega-\epsilon_{k}}=\frac{i \pi}{2 D} \sum_{\alpha} V_{\alpha}^{2}, A_{\alpha}=-\frac{\Delta_{\alpha}}{\pi} \int \frac{G_{d}^{*}\left(\omega^{\prime}\right) f_{\alpha}\left(\omega^{\prime}\right)}{\omega^{\prime}-\omega-i 0} d \omega^{\prime}$, and $B_{\alpha}=\frac{\Delta_{\alpha}}{\pi} \int \frac{f_{\alpha}\left(\omega^{\prime}\right)}{\omega^{\prime}-\omega-i 0} d \omega^{\prime}$ (where $D$ is the half-bandwidth and is taken as energy unit) the following expression is obtained :

$$
G_{d d}(\omega+i 0)=\frac{1-\frac{\leq n_{d}>}{2}+G_{d d}^{*}(\omega+i 0) \sum_{\alpha} B_{\alpha}}{\omega-E_{0}+i \Delta+\sum_{\alpha} B_{\alpha}+G_{d d}^{*}(\omega+i 0) 2 i \Delta \sum_{\alpha} B_{\alpha}} .
$$


In getting this expression we use the approximation $A_{\alpha} \approx-G_{d d}^{*} B_{\alpha}$, which was proposed first by Lacroix for the Kondo regime, and later used also in [11, 15]. Eq.13 can be easily solved. First, one conjugates the equation, and after that $G_{d}^{*}(\omega+i 0)$ is replaced in the initial formula to obtain a simple algebraic second degree equation for $G_{d}(\omega+i 0)$, only one of the solutions being physical. The solution is analytic, but is not included here because its lengthy. It is important to mention that this solution of the EOM allows to address the low temperature regime $\left(T \ll T_{K}\right)$, which is not possible with the more simple decoupling scheme in [4].

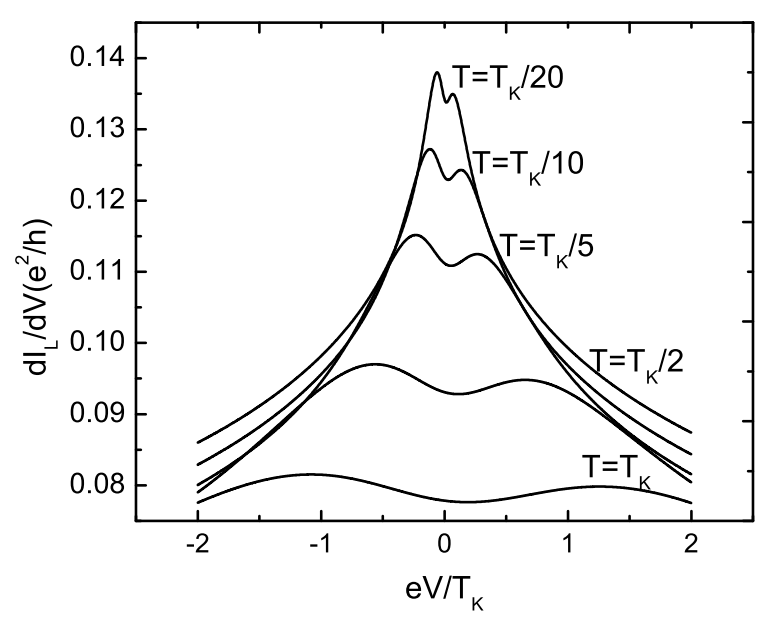

FIG. 3: Evolution of the Kondo dip with temperature; $\lambda=2$; the other parameters as in Fig.2

Fig.3 shows that the Kondo-type temperature dependance of the conductance (i.e., decrease of with increasing temperature) occurs in the range $e V / T_{K} \in[-0.5,0.5]\left(T_{k}\right.$ being calculated as in [16]). This range is in fact dependent on $\lambda$ and we checked that it becomes narrower when $\lambda$ increases.

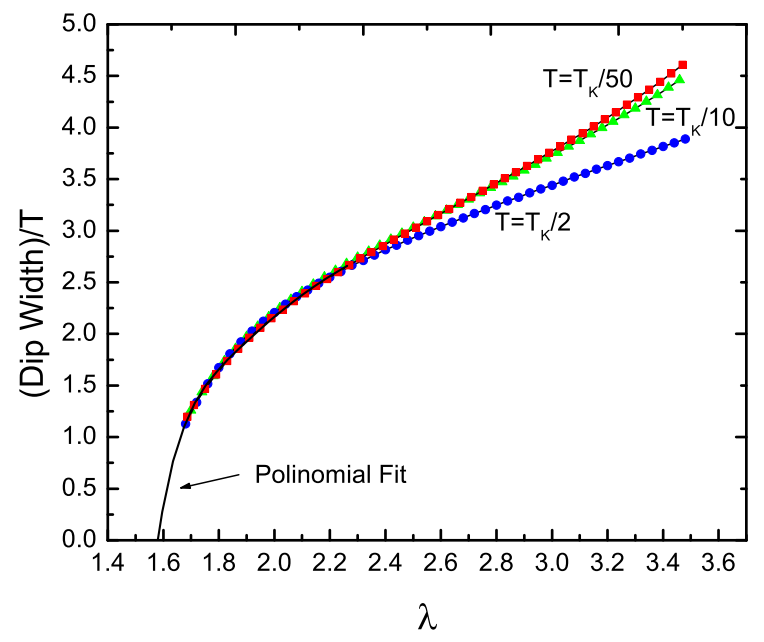

FIG. 4: The dip width scaled with temperature, as function of the asymmetry parameter $\lambda$, for three different temperatures below $T_{K}$. The linear temperature dependence is obvious for $\lambda \lesssim 2.4$, where the three scaled curves coincide. The dip vanishes at $\lambda_{0}=1.58$ which can be obtained by extrapolation.

One can also notice that the width of the dip increases with the temperature. Actually the temperature dependance of the conductance comes from the spectral function $\operatorname{Im}_{d d}(V=0, T)$. Let us define the width of the dip $w(\lambda, T)$ 
as the distance between the two peaks. By scaling the width with temperature the data from Fig.3 generates Fig.4 which shows that, for not too large values of the parameter $\lambda$, the width behaves like:

$$
w(\lambda, T)=f(\lambda) T, \text { with } \quad f\left(\lambda=\lambda_{0}\right)=0 .
$$

For $\lambda$ close to $\lambda_{0}$ the error in the calculation of the dip width becomes large, and we suggest a numerical fit to obtain the dip-peak crossover point $\lambda_{0}$. The polynomial fit gives a value of $\lambda_{0}$ very close to the exact result $\lambda_{0}=1.58$.

\section{CONCLUSIONS}

In conclusion, we have studied the non-equilibrium transport through a quantum dot connected to three reservoirs. In Kondo regime, if the potentials are applied asymmetrically on the leads (i.e. $V_{R}=\lambda V_{L}, V_{C}=0, \lambda \geq 1$ ) a peak-dip crossover of the differential conductance occurs in left lead at zero bias. The proof is based on the connection between the convexity of the differential conductance and the convexity of the density of states at $V=0$ and is independent of the Hamiltonian model. The ratios of the lead-dot couplings $\left(\Delta_{L} / \Delta_{C}, \Delta_{R} / \Delta_{C}\right)$ can be obtained if the crossover value $\lambda_{0}$ is determined experimentally.

\section{Acknowledgments}

We acknowledge support from CEEX-Research Programme and Sonderforschungsbereich 608 at the Institute of Theoretical Physics, University of Cologne.

[1] B. I. Shklovskii and A. L. Efros,Electronic Properties of Doped Semiconductors, Springer Verlag (1984), p. 228.

[2] B. L. Altshuler and A. G. Aronov, Electron-Electron Interaction In Disordered Conductors, Elsevier Science Publishers B. V., (1985), p. 46.

[3] E. Lebanon and A. Schiller, Phys. Rev. B 65, 035308 (2001).

[4] Q.-f. Sun, H. Guo, Phys. Rev. B 64, 153306 (2001).

[5] N. Shah and A. Rosch, Phys. Rev. B 73, 081309 (2005).

[6] R. Leturcq, L. Schmid, K. Ensslin, Y. Meir, D.C. Driscoll, and A.C. Gossard, Phys. Rel. Lett. 95, 126603 (2005).

[7] R. Leturcq, L. Schmid, K. Ensslin, D.C. Driscoll, and A.C. Gossard, pss-b 243, 3653 (2006).

[8] R. M. Potok, I. G. Rau, Hadas Shtrikman, Yuval Oreg, D. Goldhaber-Gordon Nature 446, 167 (2007).

[9] C. Lacroix, J. Phys. F: Metal Phys. 11, 2389 (1981).

[10] R. Swirkowicz, J. Barnas, and M. Wilczynski, Phys. Rev. B 68, 195318 (2003).

[11] I.V. Dinu, M. Ţolea, A. Aldea, Phys. Rev. B 76, 113302 (2007).

[12] R.A. Zak, K. Flensberg, Phys. Rev. B 77, 045329 (2008).

[13] A.-P. Jauho, N. S. Wingreen, Y. Meir, Phys. Rev. B 50, 5528 (1994).

[14] B. R. Bułka and P. Stefański, Phys. Rev. Lett. 86, 5128 (2001).

[15] O. Entin-Wohlman, A. Aharony, Y. Meir, Phys. Rev.B 71, 035333 (2005).

[16] F. D. M. Haldane, Phys. Rev. Lett. 40, 416 (1978). 